\title{
ELECTROMAGNETIC CALORIMETER FOR HADES EXPERIMENT AT FAIR
}

\author{
A.KUGLER ${ }^{1 a}$, C. BLUME ${ }^{g}$, W. CZYŽYCKI ${ }^{c}$, E. EPPLE $^{\text {h }}$, L. FABBIETTI ${ }^{\text {h }}$, \\ T.GALATYUK ${ }^{f}, M$. GOLUBEVA', F. GUBER', S. HLAVÁČ ${ }^{b}$, A. IVASHKIN ${ }^{i}$, \\ M. KAJETANOWIC ${ }^{d}$, B. KARDAN ${ }^{g}$, W. KOENIG ${ }^{e}$, G.KORCYL $^{d}$, K. LAPIDUS ${ }^{h}$, \\ S.LINEV ${ }^{\mathrm{e}}$, E. LISOWSKI ${ }^{\mathrm{c}}$, O.PETUKHOV ${ }^{\mathrm{i}}$, J. PIETRASZKO ${ }^{\mathrm{e}}, \mathrm{P}^{\text {P. RAMOS }}{ }^{\mathrm{a}}, \mathrm{A}$. \\ RESHETIN', A. ROST ${ }^{f}$, P. SALABURA ${ }^{d}$, Y. G. SOBOLEV ${ }^{a}$, O. SVOBODA ${ }^{a}$, P. \\ TLUSTY $^{\mathrm{a}}$, M. TRAXLER ${ }^{\mathrm{e}}$
}

${ }^{a}$ Nuclear Physics Institute, Academy of Sciences of the Czech Republic, Řež, 250 68, Czech republic

${ }^{b}$ Institute of Physics, Slovak Academy of Sciences, Bratislava, Slovakia, ${ }^{c}$ Institute of Technology Cracow, Poland

${ }^{d}$ Smoluchowski Institute of Physics, Jagiellonian University of Kraków, Cracow, Poland,

${ }^{e}$ GSI Helmholtzzentrum für Schwerionenforschung GmbH, 64291 Darmstadt, Germany ${ }^{f}$ Technische Universität Darmstadt, 64289 Darmstadt, Germany,

${ }^{g}$ Institut für Kernphysik, Goethe-Universität, 60438 Frankfurt, Germany

${ }^{h}$ Excellence Cluster 'Origin and Structure of the Universe', Technische Universität München, 85748 Garching, Germany

iInstitute for Nuclear Research, Russian Academy of Sciences, 117312 Moscow, Russia

for the HADES collaboration

Electromagnetic calorimeter (ECAL) is being developed to complement the dilepton spectrometer HADES currently operating at GSI Darmstadt, Germany. ECAL will enable the HADES@FAIR experiment to measure data on neutral meson production in heavy ion collisions at the energy range of 2-10 A GeV on the beam of future accelerator SIS100@FAIR. The calorimeter will also improve the electron-hadron separation and will as well be used for the detection of photons from strange resonances in elementary and heavy ion reactions. Calorimeter modules constructed of lead glass Cherenkov counter, photomultiplier, HV divider and optical fiber are described in the detail. Two prototypes of novel front-end electronics based on TRB3 are presented. A dedicated LED based system being developed to monitor the stability of the calorimeter during beam time is introduced as well.

52 International Winter Meeting on Nuclear Physics (Bormio 2014)

January 27 - 312014

Bormio, Italy

1

Speaker,E-mail:kugler@ujf.cas.cz 


\section{Introduction}

The High-Acceptance Di-Electron Spectrometer (HADES) experiment at GSI performs an intensive program of dielectron emission studies in few-GeV nucleon-nucleon $[1,2]$, protonnucleus [3], and nucleus-nucleus collisions [4]. Spectrometer consists of a diamond START detector, a Ring Imaging Cherenkov (RICH), four sets of Multiwire Drift Chambers (MDC), a superconducting toroidal magnet, a scintillator based time-of-flight wall (TOF) and the RPC wall built from resistive plate chambers, and a Pre-Shower detector. Spectrometer is divided into six sectors covering polar angle $18^{\circ}<\theta<85^{\circ}$, for details see [5].

Electromagnetic calorimeter (ECAL) will be located at forward angles $\left(12^{\circ}<\theta<45^{\circ}\right)$, replacing the HADES Pre-Shower detector. It will allow measurements of meson production cross-sections by detecting the gamma pair from its decay. These data are yet unknown and they will allow one to properly account for corresponding dilepton yield from Dalitz decay of neutral mesons and hence reveal other non-trivial sources of dileptons. Combining the two photon detection in the ECAL calorimeter with a charge particle detection in HADES it will also be possible to investigate $\omega$ production via the $\pi^{0} \pi^{+} \pi^{-}$and the $\pi^{0} \mathrm{e}^{+} \mathrm{e}^{-}$decays (the latter being of importance for the still unsettled question of the $\omega$ electromagnetic transition form factor). Furthermore, photon measurements would be of large interest for the HADES strangeness program which addresses also spectroscopy of neutral $\Lambda(1405)$ and $\Sigma(1385)$ resonances in elementary and HI reactions. An additional advantage of ECAL would be the improvement of the electron/pion separation at large momenta over $400 \mathrm{MeV} / \mathrm{c}$ (at lower momenta good electron/hadron identification is provided by the RICH, RPC and TOF detectors already available in HADES).

\section{Calorimeter}

The total area of the ECAL amounts $8 \mathrm{~m}^{2}$ and covers polar angles between $12^{\circ}$ and $45^{\circ}$ with almost full azimuthal coverage. Calorimeter will consist of 978 modules divided into 6 sectors with total weight of about 15 tons. Each module, see Fig.1, consists of a lead glass Cherenkov counter, photomultiplier, HV divider and optical fiber. Lead glass is a CEREN 25 type with
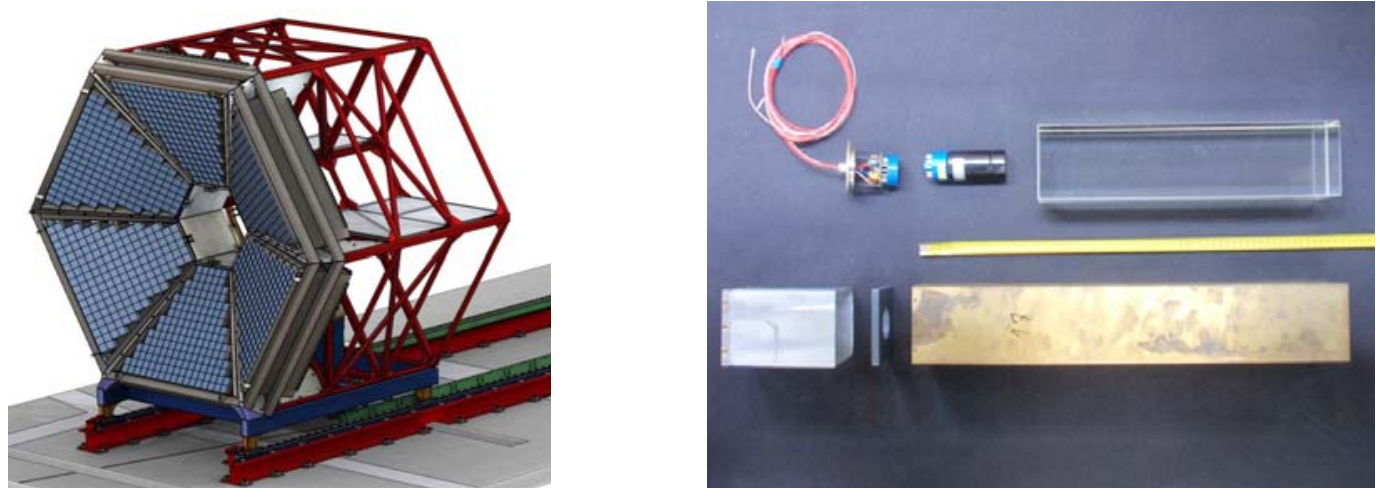

Figure 1. Schematic drawing of the ECAL detector (left) and construction parts of one module (right)

refractive index 1.708, radiation length $\mathrm{X}_{0} 2.51 \mathrm{~cm}$ and Moliére radius of $3.6 \mathrm{~cm}$. The glass was obtained on loan from the OPAL experiment. Dimensions of the crystal are $92 \times 92 \times 420 \mathrm{~mm}^{3}$ and each crystal is wrapped in white Tyvek paper (it was found out that this reflector improves the energy resolution by about $10 \%$ in comparison with aluminum or mylar reflector materials). Lead glass, photomultiplier and HV divider are housed in a brass can with $0.45 \mathrm{~mm}$ thick walls. 
A compact mechanical support structure has been designed with respect to the possibility of single module exchange without dismantling whole sector. Support structure is placed on rails using bearing guidance and $\sim 20$ tons detector can be moved along the axis of the beam only by men-power.

Three types of photomultipliers are considered at this moment. Two third of the calorimeter will use the 1.5" EMI 9903B photomultipliers from the MIRAC (WA98) detector, which passed the tests with a small size $\mathrm{Na}(\mathrm{Tl})$ scintillator and a radioactive source. Rest of the missing photomultipliers is planned to be 3" Hamamatsu R6091, 130 pieces were already bought and own design of HV divider was developed, tested and manufactured. Moreover, the 1" photomultipliers Hamamatsu R8619 are currently being tested as a price saving solution. HV power supply is based on the CAEN SY1527LC HV system with 24-channel A1535 modules providing a voltage up to $3.5 \mathrm{kV}$ and a current up to $3 \mathrm{~mA}$ per channel.

\section{Simulations}

\subsection{GEANT3 implementation}

Performance of the electromagnetic calorimeter was simulated in order to demonstrate its benefits for HADES spectrometer. Basic ECAL module geometry was implemented in the HGeant code (GEANT3-based simulation framework used by the HADES collaboration) [5], various geometries of module positions and dimensions were tested in order to find a compromise between occupancy and number of channels. No frames and support structures were implemented in the simulated geometry so far.

\subsection{Tracing of Cherenkov photons}

Simulation of the lead-glass module response was split into two parts: 1) shower development in a module (production of secondary particles and Cherenkov photons) and 2) transport of Cherenkov photons within a module (modules are optically isolated). As the transport of Cherenkov photons is a complex process involving absorption, reflection and refraction and is not described by GEANT3 correctly, a single standing code for transport of Cherenkov photons developed by M. Prokudin was used [6]. Thus, the development of an electromagnetic shower and the Cherenkov photon production is performed by GEANT3, whereas the Cherenkov photon transport is delegated to a stand-alone program. To fasten the calculation look-up table approach was used for photon transport.

\subsection{Occupancy considerations}

Occupancy of the calorimeter cells was studied on the case of $\mathrm{Ni}+\mathrm{Ni}$ collisions at $8 \mathrm{AGeV}$ and impact parameter $b<1 \mathrm{fm}$, which should be the highest possible multiplicity event studied with the HADES setup at SIS-100. Figure 2 left illustrates the event-averaged calorimeter cell occupancy using a cut on minimal deposited energy larger than $15 \mathrm{MeV}$. Squares correspond to the centers of the calorimeter modules. In the investigated conditions a highest occupancy of 0.8 is observed for the modules in the two innermost rows.

Occupancy of 0.8 might be a limiting factor of the detector and thus different geometrical arrangements are studied. One scenario is to cut the innermost modules into four separated pieces; then the cell occupancy would decrease to 0.4. Another approach can be a change of the innermost cell declination to the direction of incoming particles, primary particle cross-talk between the neighboring cells would be then minimized, see the results of modules tests with inclined photon beams in Figure 5, section 6.1. below. 


\subsection{Electron-pion separation}

The enhanced electron (positron) - pion separation at high values of particle momenta ( $>400 \mathrm{MeV} / \mathrm{c})$ will be an important benefit of the calorimeter. This topic was first studied theoretically as shown below, later proven by experiment mentioned in [7].

The $E_{E M C} / p$ quantity can be used to demonstrate electron-pion separation power. $E_{E M C} / p$ was defined as a ratio between the energy deposited by a particle in the calorimeter and the particle momentum measured by the HADES tracking system. Example of $E_{E M C} p$ ratio is presented in Figure 2 - right.
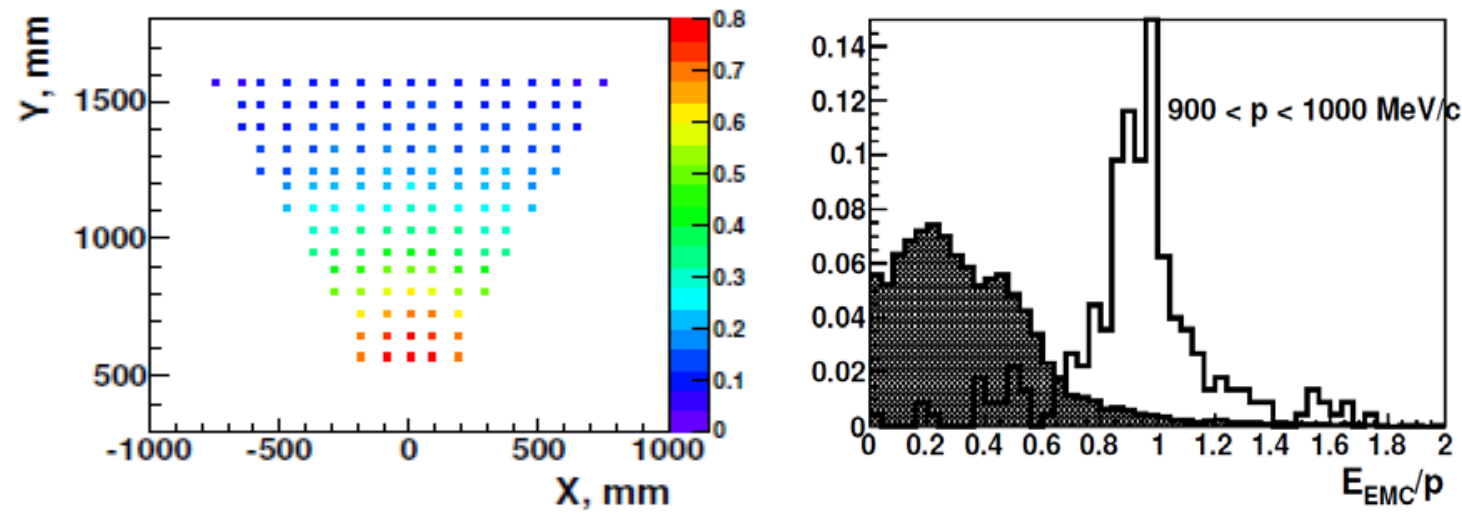

Figure 2: Average module occupancy in $\mathrm{Ni}+\mathrm{Ni}$ collisions at $8 \mathrm{AGeV}$ beam kinetic energy and impact parameter $b<1 \mathrm{fm}$ (left). $\mathrm{E}_{\mathrm{EMC}} / \mathrm{p}$ distributions for electrons (unfilled histograms) and $\pi$ - (shaded histograms) obtained at an impact parameter $b<2.5 \mathrm{fm}$ (right).

\subsection{Measurements of photon pairs}

To investigate the feasibility of the $\pi^{0}$ and $\eta$ reconstruction, events coming from the Pluto generator were processed by the reconstruction algorithm of entire HADES setup with calorimeter. Diphotons were formed taking into account all possible combinations of the identified photons. A combinatorial background was constructed with the help of the mixed event technique: uncorrelated pairs were formed taking the photons produced in different events. Afterwards, the background was normalized to the right tail of the invariant mass distribution $\left(\mathrm{M}>620 \mathrm{MeV} / \mathrm{c}^{2}\right)$. Results for $\pi^{0}$ and $\eta$ in $\mathrm{Ni}+\mathrm{Ni}$ case can be found in Figure 3.

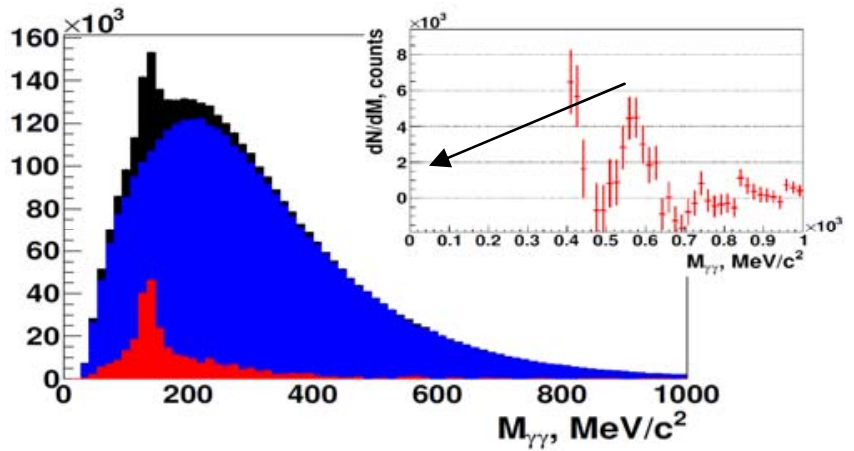

Figure 3: Diphoton invariant mass spectra reconstructed in and $N i+N i$ collisions at 8 AGeV beam kinetic energy (black histograms), combinatorial background (blue histograms) and signal after background subtraction (red histograms). $\eta$ meson signal after further analysis of the diphoton invariant mass spectra (upper right). 


\section{LED based monitoring system}

A LED based system for gain stability monitoring of the ECAL modules is being developed. It is foreseen to use blue light from a LED driven by a short signal from a pulse generator and an optical distribution system with optical fibers of CERAMOPTEC multimode type to transport the light equally to each module. Short optical fiber connects the lead glass and the rear side of the module, standard LC connector is used for connection to the light distribution system. The system is planned to be used periodically during beam times to check the time stability and thus precision of the system. A final design is not yet fixed, at present the prototyping and testing is going on.

\section{Electronics}

Two novel solutions for the read-out electronics are being developed on the principle of separate time and energy measurement. They are described in more details below.

\subsection{Cracow-design front-end board}

The 8-channels front end board based on separate time and amplitude measurement was developed at Smoluchowski Institute of Physics of Jagellonian University at Cracow. The frontend board splits the input signal from the photomultiplier into a fast and slow path. The fast path is based on a fast discriminator and delivers signal directly to the TRBv2 (Trigger and Readout Board version 2) [8] for time measurement. The slow path shapes the signal and delivers it to a fast sampling ADC addon board designed originally for HADES Pre-Shower detector. This addon board operating at $20 \mathrm{MHz}$ with 10 bit resolution is attached to TRBv2 for amplitude measurement. Thresholds can be set separately for each channel via TRB board connection. Various combinations of shaping time and gain were tested in order to reach the best energy and time resolution of the read-out system. Pulser signals with $10 \mathrm{~ns}$ width (FWHM) and $5 \mathrm{~ns}$ rise time resulted in $0.6 \%$ energy resolution and $100 \mathrm{ps}$ time resolution. Energy resolution of $3.6 \%$ and time resolution of $150 \mathrm{ps}$ were obtained using LED pulses introduced to the lead glass and measuring the response of the module.

\subsection{PaDiWa based front-end board}

Second developed scheme for preprocessing of the photomultiplier pulse is based on charge to width (Q2W) conversion. PaDiWa board (PandaDircWasa) concept is used, the front part of the board is adapted and a modified Wilkinson ADC circuit [9] is implemented in FPGA (Field Programmable Gate Array). Signal path consists basically of a capacitor, which collects the charge, and a dedicated circuit for its discharge via current source. FPGA based TDC is used to measure precisely the time to discharge. Advantage of this approach is a fast crossing of zero, and hence low dead time of the channel. In addition, this method of discharging a capacitor works as an automatic baseline restorer, since a charge equivalent to the input charge is injected with the opposite sign. Modified board was called PaDiWa AMPS and it is developed following COME\&KISS concept, thus keep it small and simple by using complex commercial elements. First simple prototype demonstrating the charge to width conversion idea was successfully tested, see section 6.1 below.

\subsection{TRB board}

The general purpose Trigger and Readout Board (TRB) version 3 [10] was developed for HADES and PANDA experiments (Barrel/DISC-DIRC and Straws) and CBM experiment 
(RICH, TOF, MVD readout) and is now foreseen as the digitizing board also for the calorimeter. A high rate, multi-hit TDC providing superior performance in terms of time precision $(<20$ ps RMS between two channels) will be used for the time measurement. The main functionality of the TRBv3 is the high precision TDC realized in FPGAs. The board also provides high bandwidth DAQ functionality with data transfer capabilities up to several hundred MByte/s and can serve up to 256 detector channels. The digitizing board controls the parameters set on the front-end boards by separate slow control lines. Implementation of TRB based DAQ to HADES network has already been done, so it can be reused for the calorimeter.

\section{Tests of modules}

Selected ECAL module prototypes were tested in various configurations to reveal their energy resolution as well as other properties.

\subsection{Tests exploiting photon beams}

Energy resolution of five modules equipped with three types of photomultipliers was measured using $0-1500 \mathrm{MeV} \gamma$-beam from MAMI facility at Mainz. Both versions of read-out electronics were used. The detectors were hit in the center by the photon beam with $6 \mathrm{~mm}$ diameter produced by scattered electrons; energy of the photon was deduced by the method of tagged electron, see table.

\begin{tabular}{|c|c|c|c|c|}
\hline Tagger channel (from1) & $E_{g}$ Low & $E_{g}$ High & $E_{g}$ Mean & $E_{g}$ Bite \\
\hline 2 & 1398.322 & 1400.327 & $\mathbf{1 3 9 9 . 3 2 5}$ & 2.005 \\
\hline 66 & 1216.127 & 1219.411 & $\mathbf{1 2 1 7 . 7 6 9}$ & 3.284 \\
\hline 121 & 1030.452 & 1034.414 & $\mathbf{1 0 3 2 . 4 3 3}$ & 3.962 \\
\hline 170 & 841.208 & 845.549 & 843.379 & 4.341 \\
\hline 210 & 675.710 & 680.453 & 678.081 & 4.743 \\
\hline 261 & 458.898 & 463.720 & 461.309 & 4.821 \\
\hline 306 & 268.440 & 273.348 & 270.894 & 4.907 \\
\hline 352 & 78.992 & 83.746 & 81.369 & 4.754 \\
\hline
\end{tabular}

Eight different photon energy bins were extracted from the tagger ranging from $72 \mathrm{MeV}$ up to $1399 \mathrm{MeV}$. Energy resolution observed changed from $20.7 \%$ at the lowest energy up to $5.5 \%$ at the highest energy, see Figure 4. Observed energy resolution did not depend on read-out electronics choice, i.e. both Cracow and PaDiWa front-end boards gave the same results, therefore we present here data for 1" photomultipliers Hamamatsu R8619 (MH5-black points), 1.5" EMI 9903B photomultipliers (EMI -red points) and 3" Hamamatsu R6091(VH3 -green points). While the resolution achieved with 1 " photomultipliers is too bad, the other two choices gave comparable results. 


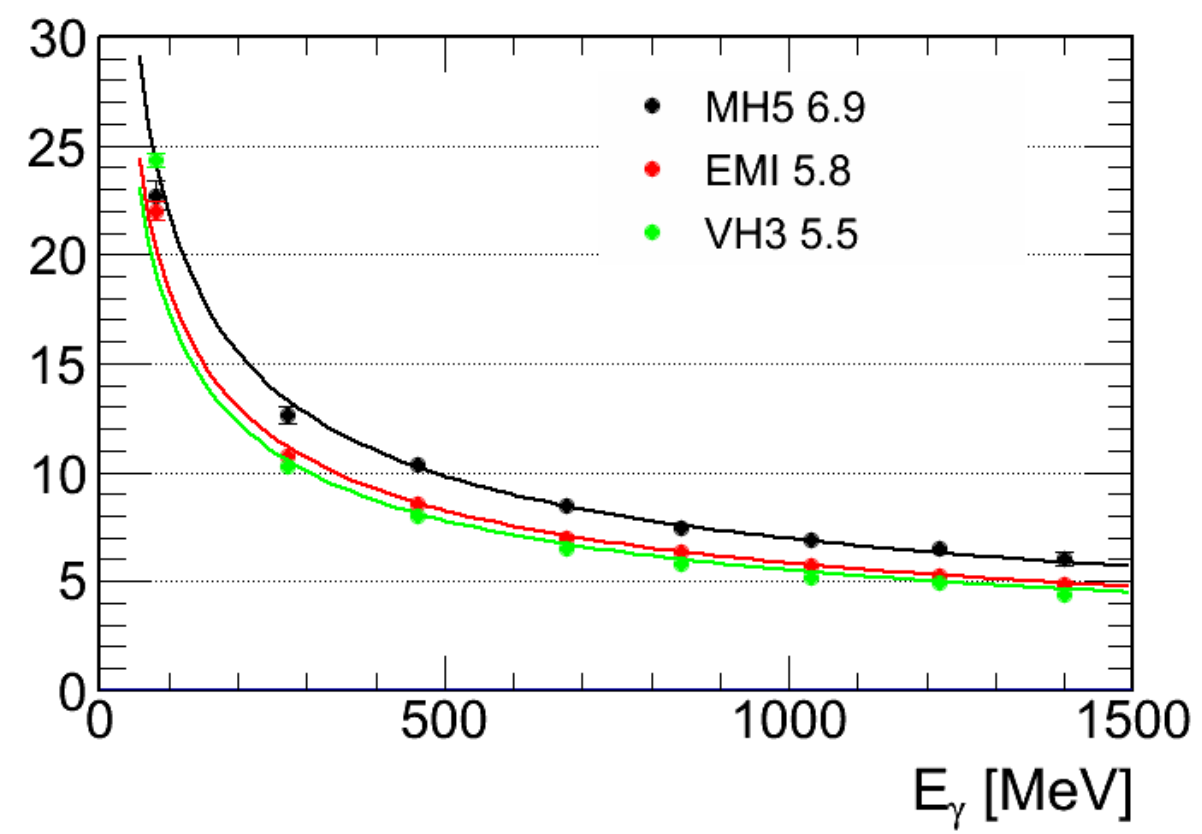

Figure 4: Energy resolution of ECAL module measured for different photon energies. Indicated are different photomultipleiers used.

Further we also studied energy leakage to neighbor module exploiting pair of two identical ECAL modules equipped with 3" Hamamatsu R6091 photomultipliers, see Figure 5. Photon beams with eight different energies, see above, and diameter $6 \mathrm{~mm}$, hitted VH3 module about $27 \mathrm{~mm}$ from the junction of modules either perpendicularly to its surface (left panel) or inclined by 12 degrees from vertical (right panel). While for the first case almost no leakage of photon energy is observed, it is quite significant for inclined photons.
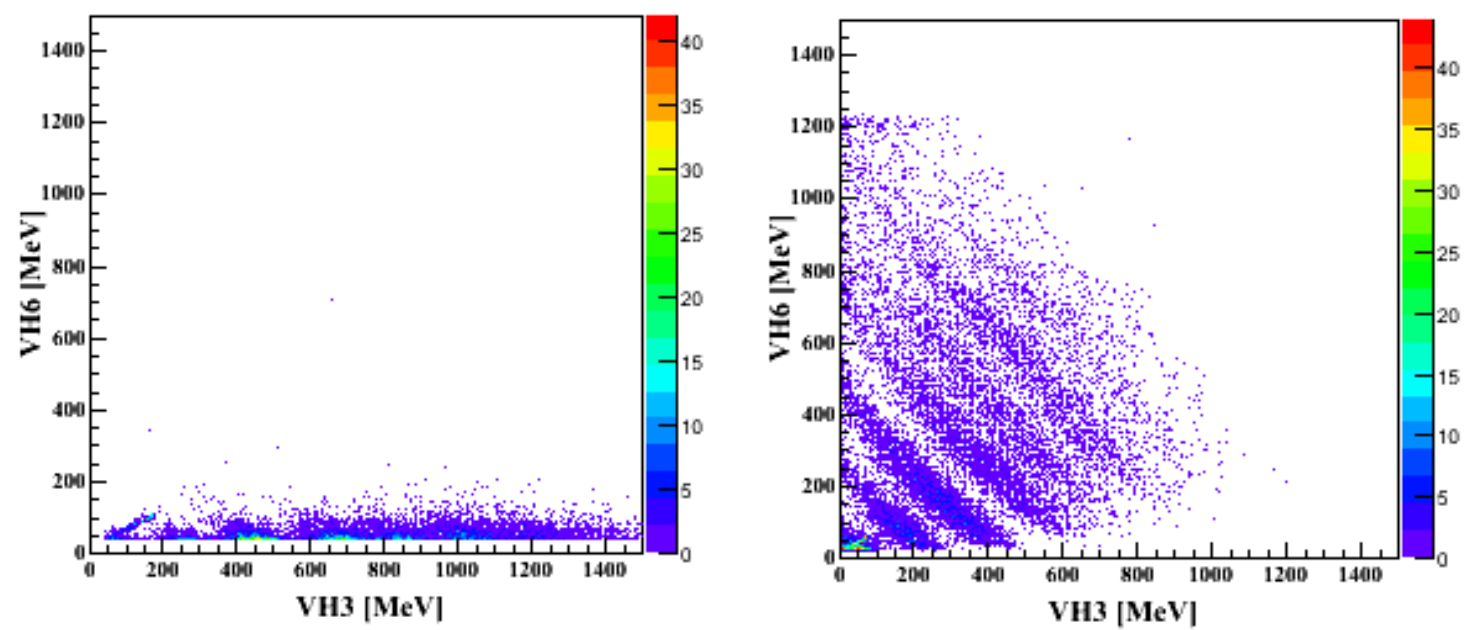

Figure 5: Distribution of energy left in pair of identical ECAL modulesVH6 and VH3 (hitted by photon). 


\subsection{Test exploiting electron/pion beam}

Dedicated measurement at CERN was carried out to evaluate electron/pion separation power and to assess time resolution, which is essential for removing hits caused by neutrons. Five identical modules with EMI photomultiplier were irradiated by secondary $\pi^{-} / \mathrm{e}^{-}$beam at T10 test beam line of the PS synchrotron. The electron fraction was $\sim 10 \%$ at $2 \mathrm{GeV} / \mathrm{c}$ and increased to about $50 \%$ at the lowest momenta $0.4 \mathrm{GeV} / \mathrm{c}$. The electron identification was provided by a $2 \mathrm{~m}$ long air Cherenkov threshold detector placed in the beam about $120 \mathrm{~cm}$ in front of the lead glass modules. Plastic scintillator of $4 \times 4 \mathrm{~cm}^{2}$ was used to produce the trigger signal for the DAQ and to select events only in the central part of the module.

The results, see Fig.6., show that the electromagnetic calorimeter can be used for an efficient $\pi^{-} / \mathrm{e}^{-}$separation at higher momenta $(\mathrm{p}>0.5 \mathrm{GeV} / \mathrm{c})$ where the time of flight cannot be used. The measured time resolution of 215 ps (sigma) will enable to remove efficiently neutrons which are not detected by the tracking system.

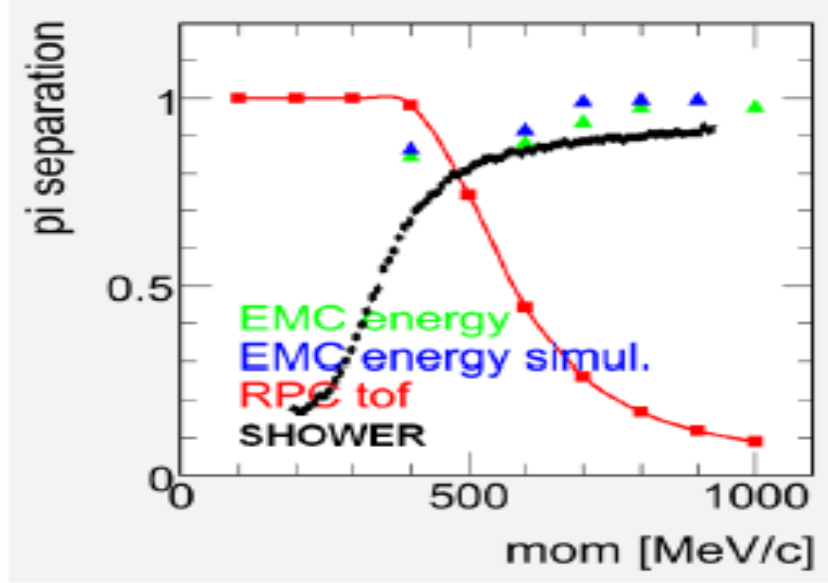

Figure 6. Electron/pion separation

\section{Summary}

Electromagnetic calorimeter for HADES spectrometer is being built at GSI, Germany. Calorimeter will extend the experimental possibilities of the HADES spectrometer by measuring meson production cross-sections using the gamma pair from its decay and will offer enhanced electron/pion separation at large momenta over $400 \mathrm{MeV} / \mathrm{c}$.

Calorimeter modules of lead glass, photomultiplier with HV divider and optical fiber are being assembled and technical solutions are tested with respect to the achieved energy resolution. LED based system for stability monitoring has been prototyped giving the same detector response as real signals from cosmic muons.

Novel front-end electronic solution is being developed using separate TDC and ADC measurement. Two approaches based on fast sampling ADC and charge measurement are investigated. 


\section{Acknowledgments}

We would like to express special thanks to A. Neiser, P. Ott, P. Otte, A. Thomas, P. Skott, and A. Neiser from Institut für Kernphysik, Johannes Gutenberg-Universität, Mainz, Germany, for their help with the measurements and to the staff of the MAMI facility for providing excellent beam conditions. This work was supported by Czech MSMT LG 120007, GACR 13-067595 and AS CR M100481202.

\section{References}

[1] G. Agakishiev et al.(HADES Collaboration), Phys. Rev. C85, 054005 (2012).

[2] G. Agakishiev et al. (HADES Collaboration), Eur. Phys. J. A48, 64 (2012).

[3] G. Agakishiev et al. (HADES Collaboration), Phys. Lett. B715, 304 (2012).

[4] G. Agakishiev et al. (HADES Collaboration), Phys. Rev. C84, 014902 (2011).

[5] G. Agakishiev et al. (HADES Collaboration), Eur. Phys. J. A 41, 243 (2009).

[6] V. Arefev et al., A study of light collection in "Shashlik" calorimeters, Instrum. Exp. Tech. 51, p. 511 (2008).

[7] W. Czyzycki et al., Electromagnetic Calorimeter for HADES (Technical Design Report), arXiv:1109.5550 [nucl-ex].

[8] Frohlich et al., A General Purpose Trigger and Readout Board for HADES and FAIRExperiments, IEEE Trans.Nucl.Sci., 55:59-66 (2008).

[9] D. Ponikvar, Wilkinson-type ADC with short conversion time and low clock frequency, NIM A 408(2-3):523 - 529 (1998).

[10] J. Michel et al., JINST 8, C02034 (2013) 